\title{
Measurement of the energy dependence of the form factor $f_{+}$ in $\mathrm{K}_{\mathrm{e} 3}^{0}$ decay
}

\section{CPLEAR Collaboration}

A. Apostolakis ${ }^{\text {a }}$, E. Aslanides ${ }^{k}$, G. Backenstoss ${ }^{\text {b }}$, P. Bargassa ${ }^{\mathrm{m}}$, O. Behnke ${ }^{\mathrm{q}}$, A. Benelli ${ }^{\text {b }}$, V. Bertin ${ }^{\mathrm{k}}, \mathrm{F}$. Blanc ${ }^{\mathrm{g}, \mathrm{m}}, \mathrm{P}$. Bloch ${ }^{\mathrm{d}}$, P. Carlson ${ }^{\mathrm{o}}, \mathrm{M}$. Carroll ${ }^{\mathrm{i}}$, E. Cawley ${ }^{\mathrm{i}}$, M.B. Chertok ${ }^{\mathrm{c}}$, M. Danielsson ${ }^{\mathrm{o}}$, M. Dejardin ${ }^{\mathrm{n}}$, J. Derre ${ }^{\mathrm{n}}$, A. Ealet ${ }^{\mathrm{k}}$, C. Eleftheriadis ${ }^{\text {p }, \text { W. Fetscher }}{ }^{\mathrm{q}}$, M. Fidecaro ${ }^{\mathrm{d}}$, A. Filipčič ${ }^{j}$, D. Francis ${ }^{\mathrm{c}}$, J. Fry ${ }^{\mathrm{i}}$, E. Gabathuler ${ }^{\text {i }}$, R. Gamet ${ }^{\text {i }}$, H.-J. Gerber ${ }^{\text {q }}$, A. Go ${ }^{\text {d }}$, A. Haselden ${ }^{\text {i }, ~ P . J . ~ H a y m a n ~}{ }^{\text {i, }}$, F. Henry-Couannier ${ }^{k}$, R.W. Hollander ${ }^{\mathrm{f}}$, K. Jon-And ${ }^{\mathrm{o}}$, P.-R. Kettle ${ }^{\mathrm{m}}$, P. Kokkas ${ }^{\mathrm{b}}$, R. Kreuger ${ }^{\text {f }}$, R. Le Gac ${ }^{\text {k}}$, F. Leimgruber ${ }^{\text {b }}$, I. Mandić ${ }^{j}$, N. Manthos ${ }^{\text {h }}$, G. Marel ${ }^{\text {n, }}$, M. Mikuž ${ }^{\mathrm{j}}$, J. Miller ${ }^{\mathrm{c}}$, F. Montanet ${ }^{\mathrm{k}}$, A. Muller ${ }^{\mathrm{n}}$, T. Nakada ${ }^{\mathrm{m}}$, B. Pagels ${ }^{\mathrm{q}}$, I. Papadopoulos ${ }^{\text {p }}$, P. Pavlopoulos ${ }^{\text {b }}$, G. Polivka ${ }^{\text {b }}$, R. Rickenbach ${ }^{\text {b }}$, B.L. Roberts ${ }^{\mathrm{c}}$, T. Ruf ${ }^{d}$, M. Schäfer ${ }^{\mathrm{q}}$, L.A. Schaller ${ }^{\mathrm{g}}$, T. Schietinger ${ }^{\mathrm{b}}$, A. Schopper ${ }^{\mathrm{d}}$, L. Tauscher ${ }^{\text {b }}$, C. Thibault ${ }^{1}$, F. Touchard ${ }^{k}$, C. Touramanis i , C.W.E. Van Eijk ${ }^{\text {f }}$, S. Vlachos ${ }^{\text {b }}$, P. Weber ${ }^{\mathrm{q}}$, O. Wigger ${ }^{\mathrm{m}}$, M. Wolter ${ }^{\mathrm{q}}$, D. Zavrtanik ${ }^{\mathrm{j}}$, D. Zimmerman ${ }^{\mathrm{c}}$

\author{
${ }^{\text {a }}$ University of Athens, Greece \\ ${ }^{\mathrm{b}}$ University of Basle, Switzerland \\ ${ }^{\mathrm{c}}$ Boston University, USA \\ d CERN, Geneva, Switzerland \\ ${ }^{\mathrm{e}}$ LIP and University of Coimbra, Portugal \\ ${ }^{\mathrm{f}}$ Delft University of Technology, Netherlands \\ ${ }^{\mathrm{g}}$ University of Fribourg, Switzerland \\ ${ }^{\mathrm{h}}$ University of Ioannina, Greece \\ ${ }^{\mathrm{i}}$ University of Liverpool, $U K$ \\ ${ }^{j}$ J. Stefan Inst. and Phys. Dep., University of Ljubljana, Slovenia \\ ${ }^{\mathrm{k}}$ CPPM, IN2P3-CNRS et Université d'Aix-Marseille II, France \\ ${ }^{1}$ CSNSM, IN2P3-CNRS, Orsay, France \\ ${ }^{\mathrm{m}}$ Paul Scherrer Institut (PSI), Switzerland \\ ${ }^{n}$ CEA, DSM / DAPNIA, CE-Saclay, France \\ ${ }^{\circ}$ Royal Institute of Technology, Stockholm, Sweden \\ ${ }^{\mathrm{p}}$ University of Thessaloniki, Greece \\ ${ }^{\mathrm{q}}$ ETH-IPP Zürich, Switzerland
}




\section{Abstract}

Neutral-kaon decays to $\pi \mathrm{e} \nu$ were analysed to determine the $q^{2}$ dependence of the $\mathrm{K}_{\mathrm{e} 3}^{0}$ electroweak form factor $f_{+}$. Based on 365612 events, this form factor was found to have a linear dependence on $q^{2}$ with a slope $\lambda_{+}=0.0245 \pm$ $0.0012_{\text {stat }} \pm 0.0022_{\text {syst }}$. (C) 2000 Published by Elsevier Science B.V. All rights reserved.

\section{Introduction}

The form factor $f_{+}$of neutral-kaon decay to $\pi \mathrm{e} \nu$ and its energy dependence have a rather long history [1,2]. The Particle Data Group [2] lists 19 different measurements for the slope parameter $\lambda_{+}$, ranging from values compatible with zero up to 0.045 , all with rather large error bars. Also, the theoretical values scatter between 0.018 and 0.033 [3]. In order to clarify this situation, a subsample of the neutralkaon decays to $\pi \mathrm{e} \nu$ recorded in the CPLEAR experiment [4-6] was analysed to obtain with large statistics another independent and possibly more accurate determination of the parameter $\lambda_{+}$. The results of this analysis are reported in the present paper.

\section{Phenomenology of $K_{\mathrm{e} 3}^{0}$ decay}

Assuming V-A coupling, the neutral-kaon decays to $\pi \mathrm{e} \nu$ are pure vector transitions. The matrix element can then be written as

$$
\begin{aligned}
\mathscr{M}= & \frac{G}{\sqrt{2}} V_{\text {us }}\left[f_{+}\left(q^{2}\right)\left(p_{\mathrm{K}}+p_{\pi}\right)^{\mu}\right. \\
& \left.+f_{-}\left(q^{2}\right)\left(p_{\mathrm{K}}-p_{\pi}\right)^{\mu}\right] \bar{U}_{\mathrm{e}} \gamma_{\mu}\left(1+\gamma_{5}\right) U_{\nu},
\end{aligned}
$$

where $G$ is the Fermi constant, $V_{\text {us }}$ the CKM matrix element coupling the $\mathrm{u}$ - and s-quarks, $f_{+}$and $f_{-}$are the vector form factors, $\bar{U}_{\mathrm{e}}$ and $U_{\nu}$ the lepton spinors, $p_{\mathrm{K}}$ and $p_{\pi}$ the kaon and the pion four-momenta, respectively, and $q=p_{\mathrm{K}}-p_{\pi}$ is the four-momentum transfer to the e $\nu$ pair. Experimentally, the fourmomentum squared $q^{2}$, on which $f_{+}$and $f_{-}$depend, is determined by the neutral-kaon momentum
$P_{\mathrm{K}}$, the pion momentum $P_{\pi}$ and the cosine of the angle between these two particles $\cos \left(\alpha_{\mathrm{K} \pi}\right)$ :

$$
\begin{aligned}
q^{2}= & \left(p_{\mathrm{K}}-p_{\pi}\right)^{2} \\
= & m_{\mathrm{K}}^{2}+m_{\pi}^{2}-2 \sqrt{m_{\mathrm{K}}^{2}+P_{\mathrm{K}}^{2}} \sqrt{m_{\pi}^{2}+P_{\pi}^{2}} \\
& +2 P_{\mathrm{K}} P_{\pi} \cos \left(\alpha_{\mathrm{K} \pi}\right),
\end{aligned}
$$

where $m_{\mathrm{K}}$ and $m_{\pi}$ are the neutral-kaon and the charged-pion mass, respectively. From Eq. (1), the density $\rho$ of the Dalitz plot distribution can be deduced:

$\rho \propto f_{+}^{2}\left(q^{2}\right)$.

In Eq. (3) the terms containing $f_{-}\left(q^{2}\right)$ were neglected owing to the small electron mass $m_{\mathrm{e}}$ [1]. The form factor $f_{+}$can be measured from the $q^{2}$ distribution, in the physical range

$m_{\mathrm{e}}^{2}<q^{2}<\left(m_{\mathrm{K}}-m_{\pi}\right)^{2}$.

The $q^{2}$ dependence of $f_{+}\left(q^{2}\right)$ is usually approximated as

$f_{+}\left(q^{2}\right)=f_{+}(0)\left(1+\lambda_{+} \frac{q^{2}}{m_{\pi}^{2}}\right)$,

where $\lambda_{+}$is the linear energy dependence parameter [2]. The experimental method for the determination of $\lambda_{+}$consists in comparing the measured $q^{2}$ distribution with that of a sample simulated using a constant form factor $\left(\lambda_{+}=0\right)$.

\section{The CPLEAR experiment}

The neutral kaons were produced in $\overline{\mathrm{p}} \mathrm{p}$ annihilations to $\mathrm{K}^{-} \pi^{+} \mathrm{K}^{0}$ and $\mathrm{K}^{+} \pi^{-} \overline{\mathrm{K}}^{0}$, each channel corresponding to a branching fraction of $\approx 0.2 \%$. The $200 \mathrm{MeV} / c$ antiprotons, provided at a rate of $1 \mathrm{MHz}$ by the Low Energy Antiproton Ring (LEAR) at 
CERN, were slowed down and annihilated at rest in a high-pressure (26 bar) gaseous hydrogen target situated at the centre of the CPLEAR detector [7]. This detector was designed to measure the charged $\mathrm{K} \pi$ pair produced together with the neutral kaon as well as the particles resulting from the subsequent neutral-kaon decay.

The detector had a cylindrical geometry around the beam direction and was situated inside a solenoid magnet that produced a field of $0.44 \mathrm{~T}$. The tracking of the charged particles was performed with two layers of proportional chambers, six layers of drift chambers and two layers of streamer tubes. The charged-particle identification detector (PID) consisted of a threshold Cherenkov counter sandwiched between two scintillator counters, providing energyloss, Cherenkov-light and time-of-flight information. An electromagnetic calorimeter completed the set-up. A dedicated hardwired multilevel trigger performed an efficient online event selection, which was needed owing to the small branching fraction of the neutralkaon production channels. The trigger was based on particle identification, event kinematics and shower counting. It allowed an online selection of the desired annihilation channels, while the decay channels were mainly selected offline.

\section{Event selection}

The $\bar{p}$-annihilation events containing, together with a $\mathrm{K}^{\mp} \pi^{ \pm}$pair, a neutral kaon which subsequently decays to $\pi \mathrm{e} \nu$, are first selected by requiring that the events have four charged tracks with zero total charge - two of which (primaries) originate from the annihilation vertex inside the target and two (secondaries) from a well-separated decay vertex - and by identifying in the PID one of the decay tracks as an electron or a positron, and one of the primaries as the charged kaon. The electron-pion separation is performed by a neural network using momentum, energy-loss, Cherenkov-light and timeof-flight information. The average efficiency of the electron identification is $85 \%$, with $2 \%$ pion contamination [4]. In addition we require a total momentum $>350 \mathrm{MeV} / c$ for the charged kaon, momenta in the range $80-1000 \mathrm{MeV} / c$ for the primary and sec- ondary pions, and a momentum in the range 50 $350 \mathrm{MeV} / c$ for the electron (see also Ref. [4]).

Finally, a constrained fit (6C) was used. The fit includes kinematic constraints (imposing fourmomentum conservation at both the annihilation and the decay vertices), and geometric constraints (requiring each track to originate from either the annihilation or the decay vertex, and the $\mathrm{K}^{0}$ momentum to be aligned to the line joining these vertices). With these selection criteria we are left with three sources of background, namely the neutral-kaon decays to $\pi \mu \nu, \pi^{+} \pi^{-}$and $\pi^{+} \pi^{-} \pi^{0}$.

A cut on the missing mass under the $\pi \mu \nu$ event hypothesis allows a significant reduction of this background which is the most important one. The $\pi^{+} \pi^{-}$events are efficiently rejected using their two-body decay properties, i.e. requiring that in the neutral-kaon rest frame the opening angle between the secondaries be $<154^{\circ}$. A further background reduction is achieved by using constrained fits under the hypothesis of $\pi^{+} \pi^{-}$decay or $\pi^{+} \pi^{-} \pi^{0}$ decay. An opening angle of $>26^{\circ}$ between the secondaries ensures that the sample is free from $\mathrm{e}^{+} \mathrm{e}^{-}$pairs originating from photon conversions. Similarly, the opening angle between the two primaries must be $<168^{\circ}$ in order to reject tracks backscattered from the external part of the detector.

\section{The data (real and Monte Carlo simulated)}

A total of 365612 events were selected from the 1995 CPLEAR data sample (real data). The $q^{2}$ distribution of the real data and the detector acceptance as a function of $q^{2}$ are shown in Fig. 1. The acceptance is practically independent of $q^{2}$ in the range $0.02-0.12 \mathrm{GeV}^{2}$.

We note here that the measurement of the $q^{2}$ dependence of the $\mathrm{K}_{\mathrm{e} 3}^{0}$ form factor relies on the comparison of the real-data distribution with the distribution simulated with a constant form factor $\left(\lambda_{+}=0\right)$. Therefore, the absolute acceptance of the detector should be well monitored and understood.

For the present measurement, the neutral-kaon decays to $\pi \mathrm{e} \nu, \pi \mu \nu, \pi^{+} \pi^{-}$and $\pi^{+} \pi^{-} \pi^{0}$ were simulated by the Monte Carlo method. These simulations included all geometrical and physical properties of the detector. Multiple scattering, energy loss, $\delta$-ray 

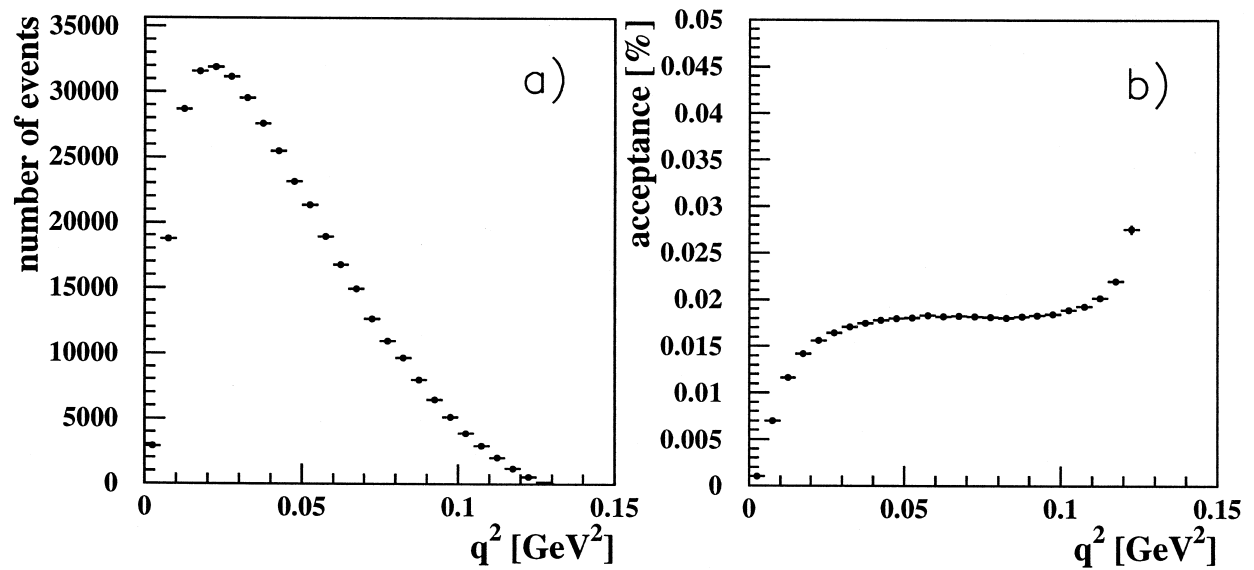

Fig. 1. a) The $q^{2}$ distribution of the real-data sample (365612 events); b) the detector (absolute) acceptance as a function of the four-momentum transfer $q^{2}$.

emissions and the random noise in the detector were also simulated. The intermediate resonances, in particular $\mathrm{K}^{ \pm *}$ and $\mathrm{K}^{0 *}$, were taken into account when simulating $\mathrm{p} \overline{\mathrm{p}}$ annihilations. The $\pi \mathrm{e} \nu$ events were generated with a form factor $f_{+}$of slope $\lambda_{+}=0.0300$, according to Ref. [2]. A weight was applied to each $\pi \mathrm{e} \nu$ simulated event, in order to restore the constant form factor needed in this analysis $\left(\lambda_{+}=0\right)$. The effect of radiative decays was not simulated, and therefore had to be corrected. The calculations of Ginsberg [9] were used to determine the weight to be applied on an event-by-event basis, resulting in an average correction of $\approx 8 \%$ in the $q^{2}$ range of interest. The generated events were then selected by a simulation of the online trigger and passed through the event selection as described previously. The final sample of simulated events is approximately ten times larger than the real-data sample, thus reducing the statistical uncertainties.

From this Monte Carlo simulation, we concluded that the real-data sample contains, for the full $q^{2}$ range, $93.1 \% \pi \mathrm{e} \nu$ events (signal), $6.2 \% \pi \mu \nu$ events (main background), $0.6 \% \pi^{+} \pi^{-}$events and only $0.1 \% \pi^{+} \pi^{-} \pi^{0}$ events. Fig. 2 shows the $q^{2}$ distributions of the $\pi \mathrm{e} \nu$ signal and the different background components, including semileptonic events for which the identifications of the electron and the pion were interchanged $(\mathrm{e} \pi \nu)$. The latter category constitutes $0.8 \%$ of the signal. Since the form factor applies to $\pi \mathrm{e} \nu$ as well as to e $\pi \nu$ decays, these events are included in the $\pi \mathrm{e} \nu$ signal for the determination of the slope parameter $\lambda_{+}$and contribute to the resolution [8].

The quality of the Monte Carlo simulation (with $\lambda_{+}=0.0300$ for the $\pi \mathrm{e} \nu$ events) was checked by comparing the different kinematical and geometrical distributions with those from the real data. Fig. 3

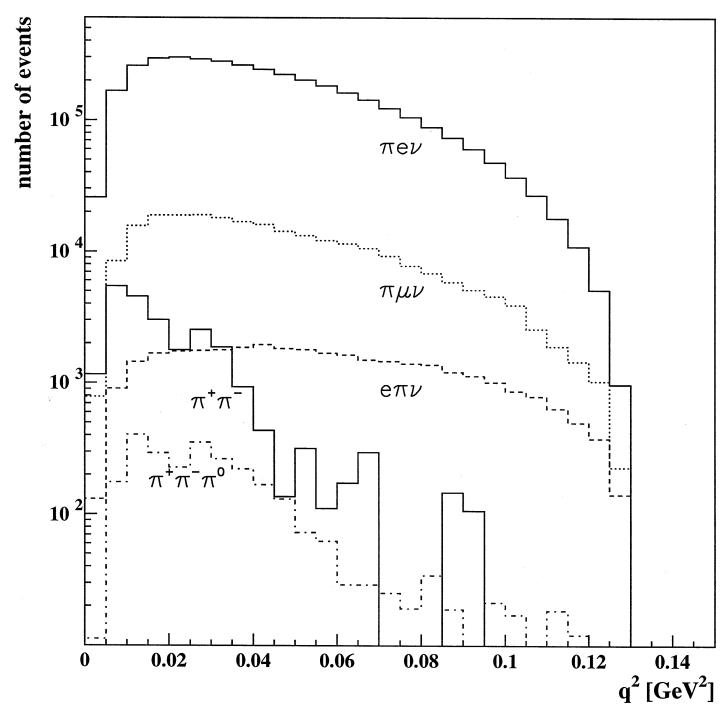

Fig. 2. The $q^{2}$ distribution of the simulated-data sample. Signal $(\pi \mathrm{e} \nu)$ and background $\left(\pi \mu \nu, \pi^{+} \pi^{-}, \pi^{+} \pi^{-} \pi^{0}\right)$ distributions are shown separately. The $\pi \mathrm{e} \nu$ distribution in the case where electron and pion were misidentified is also shown $(\mathrm{e} \pi \nu)$. 

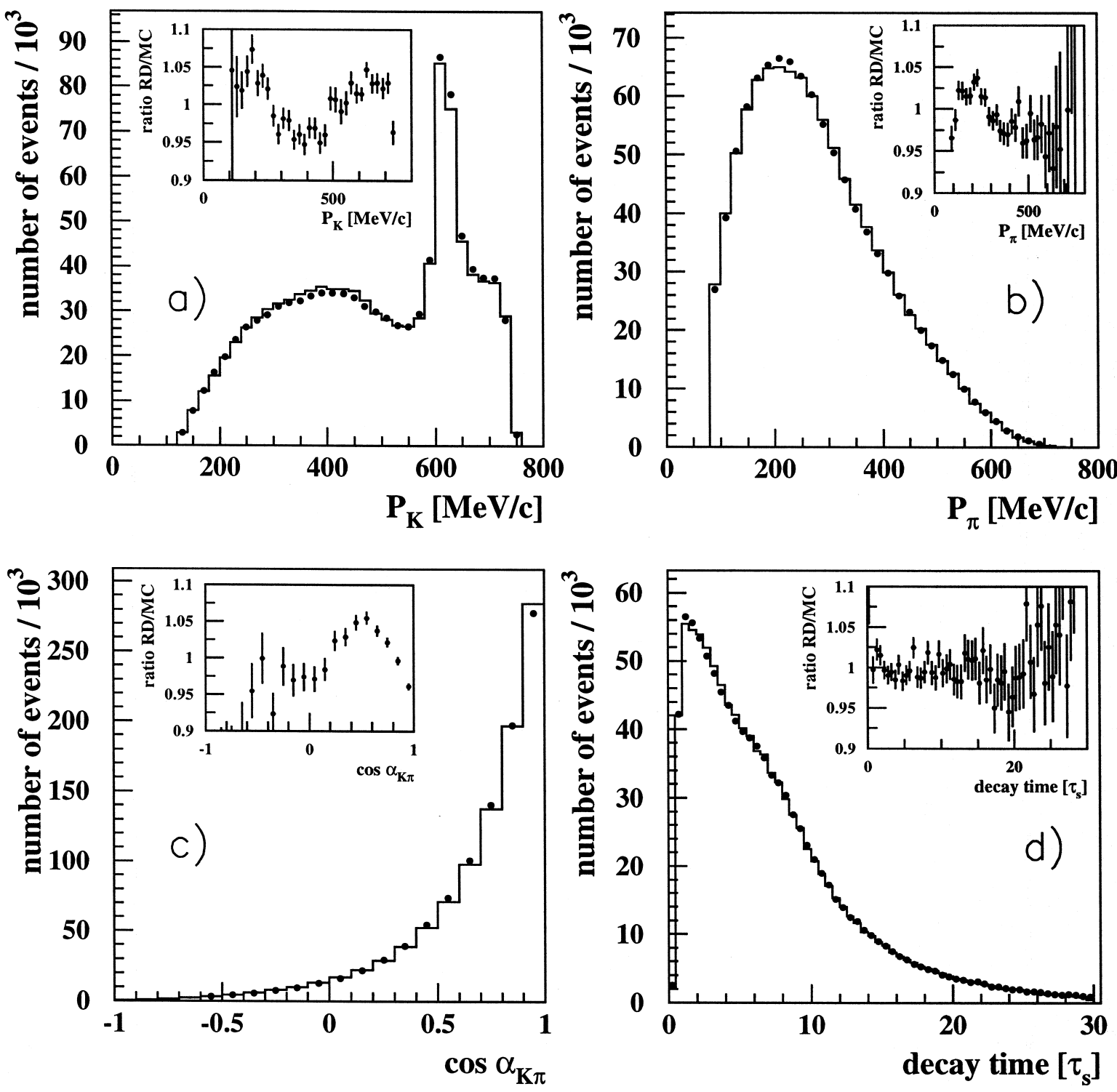

Fig. 3. The measured (dots) and simulated (solid line) distributions for four relevant variables, normalized to $10^{3}$ events: a) the neutral-kaon momentum $P_{\mathrm{K}}$, b) the secondary pion momentum $P_{\pi}$, c) the cosine of the angle between these two particles $\cos \left(\alpha_{\mathrm{K} \pi}\right)$, and d) the neutral-kaon decay time. The background contribution has been subtracted from the real-data distributions. Owing to the large statistics of the data samples, the uncertainty on each point is smaller than the dot size. For each variable, the ratio of the measured (Real Data, RD) to the simulated (MC) distributions is included in the plot.

shows the measured and simulated distributions together with their ratio, for the variables entering in the determination of $q^{2}-P_{\mathrm{K}}, P_{\pi}, \cos \left(\alpha_{\mathrm{K} \pi}\right)-$ and for the neutral-kaon decay time. The simulated kine- matical variables, i.e. the particle momenta, as well as the opening angles between particles, agree within $\pm 5 \%$ with the corresponding distributions from the real data. The geometrical variables, i.e. the primary 
and secondary vertex positions, are correctly described within $\pm 10 \%$. Finally, the neutral-kaon decay-time distribution is reproduced within $\pm 2 \%$.

\section{Results}

First, the background contribution, as determined by Monte Carlo simulation and known branching fractions [2], was subtracted from the measured $q^{2}$ distribution, to obtain the real data distribution $N_{R D}\left(q^{2}\right)$.

Due to the physical bounds of $q^{2}$ [cf. Eq. (4)], the resolution is not constant over the $q^{2}$ range and the resolution shape is asymmetric. The average $q^{2}$ resolution was determined to be $0.006 \mathrm{GeV}^{2}$. In order to fully take into account the resolution [8], we use the method outlined in the following. Three weighted distributions were determined from the simulated $\pi$ e $\nu$ events:

- $N_{1}\left(q^{2}\right)$, the number of events reconstructed in a $q^{2}$ bin,

- $N_{2}\left(q^{2}\right)$, the number of events reconstructed in a $q^{2}$ bin, each event being weighted by the second power of the generated four-momentum transfer $q_{\text {true }}^{2}$

- $N_{3}\left(q^{2}\right)$, the number of events reconstructed in a $q^{2}$ bin, each event being weighted by the fourth power of the generated four-momentum transfer $q_{\text {true }}^{4}$.

With these definitions and using Eqs. (3) and (5), for a given value of $\lambda_{+}$the expected number of events in a bin of $q^{2}$ is given by

$$
\begin{aligned}
& N_{\mathrm{MC}}\left(q^{2}\right) \\
& \quad=C\left(N_{1}\left(q^{2}\right)+2 \lambda_{+} \frac{N_{2}\left(q^{2}\right)}{m_{\pi}^{2}}+\lambda_{+}^{2} \frac{N_{3}\left(q^{2}\right)}{m_{\pi}^{4}}\right) .
\end{aligned}
$$

We performed a $\chi^{2}$-fit of Eq. (6) to the data, in the range $0.02<q^{2}<0.12 \mathrm{GeV}^{2}$, leaving $\lambda_{+}$and the normalization parameter $C$ free. The limits of the fit interval were chosen for the following reasons:

- The radiative corrections remain relatively constant in this range, thus leaving the spectrum shape unchanged.

- The acceptance is practically flat in this range (see Fig. 1).
- The $\pi^{+} \pi^{-}$background is mainly situated at $q^{2}<0.02 \mathrm{GeV}^{2}$, while the $\pi \mu \nu$ decays contribute mainly at the high $q^{2}$ values (see Fig. 2). The result of the fit, shown in Fig. 4, gives $\lambda_{+}=$ $0.0245 \pm 0.0012$, with $\chi^{2}=33.0$ for 18 degrees of freedom $(\mathrm{CL}=1.7 \%)$. The quoted uncertainty is purely statistical. This result contains the effect of the radiative corrections, which reduced the final value by 0.0010 .

To the statistical uncertainty of $5 \%$, the systematic uncertainties have to be added. Different sources of systematics were investigated:

- The different backgrounds were estimated to be known to $\pm 10 \%$. The resulting uncertainty on $\lambda_{+}$is \pm 0.0006 .

- The sensitivity of $\lambda_{+}$to the bounds of the $q^{2}$ range was studied by changing the limits. It was found that the value of $\lambda_{+}$is insensitive to both the upper and the lower bounds. Therefore, such a possible systematic contribution can be neglected.

- The quality of the simulation of the three variables used in the determination of $q^{2}$, i.e. $P_{\mathrm{K}}, P_{\pi}$ and $\cos \left(\alpha_{\mathrm{K} \pi}\right)$ [Eq. (2)], was studied quantitatively, and the systematic effect on the value of $\lambda_{+}$was determined. In the simulation sample each variable was varied by the ratio of the measured to the simulated distributions, Fig. 3. The systematic effect was estimated from the deviation of the value of $\lambda_{+}$, before and after this variation. The uncertainties obtained for the three variables (given in Table 1) are added quadratically to obtain the systematic uncertainty, neglecting their correlations.

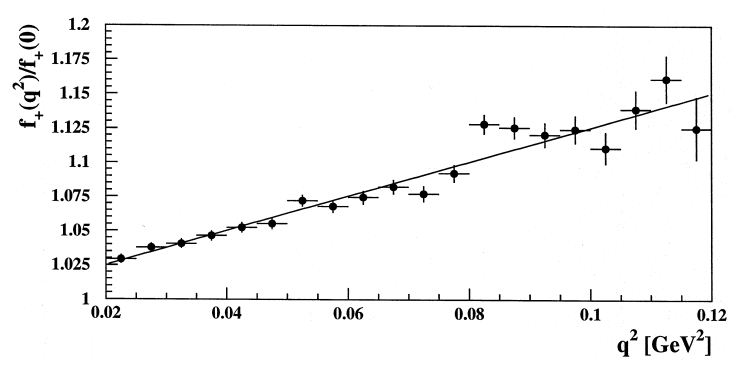

Fig. 4. The $q^{2}$ dependence of the form factor $f_{+}\left(q^{2}\right)$, normalized such that $f_{+}\left(q^{2}=0\right)=1.0$. The result of the fit (solid line) is superimposed to the data points (dots). The $q^{2}$ range of the figure corresponds to the range used in the fit. 
Table 1

Systematic uncertainties.

\begin{tabular}{ll}
\hline Source & $\Delta\left(\lambda_{+}\right)$ \\
Background level & \pm 0.0006 \\
$q^{2}$ range for the fit & Negligible \\
Quality of the simulation: & \\
$P_{\mathrm{K}}$ spectrum & \pm 0.0001 \\
$P_{\pi}$ spectrum & \pm 0.0014 \\
$\cos \left(\alpha_{\mathrm{K} \pi}\right)$ spectrum & \pm 0.0016 \\
Total & $\pm \mathbf{0 . 0 0 2 2}$ \\
\hline
\end{tabular}

- No significant difference between the $\mathrm{K}^{0}$ and the $\overline{\mathrm{K}}^{0}$ samples was found.

- The effects of $\mathrm{CP}$ violation, of $\mathrm{K}_{\mathrm{S}}$ regeneration and of the different $\mathrm{K}^{0}$ and $\overline{\mathrm{K}}^{0}$ detection efficiencies were corrected for in the observed data. These corrections had no significant effect on the measurement, as expected from their low impact on the energy distributions.

A summary of the systematic uncertainties is shown in Table 1. The total systematic uncertainty is determined to be \pm 0.0022 , and our final result is $\lambda_{+}=$ $0.0245 \pm 0.0012_{\text {stat }} \pm 0.0022_{\text {syst }}$. The large value of the systematic uncertainties originates from the high sensitivity of this measurement to the absolute identification of the electron, in contrast to other measurements performed at CPLEAR, which are only sensitive to relative effects [4-6].

The addition of a quadratic term $+\lambda_{+}^{\prime \prime} q^{4} / m_{\pi}^{4}$ to formula (5) does not improve the quality of the fit. The fitted value for $\lambda_{+}^{\prime \prime}$ is compatible with zero. Therefore, within the present experimental accuracy, there is no need for a quadratic term in the energy dependence of the $\mathrm{K}_{\mathrm{e} 3}^{0}$ form factor.

\section{Conclusion}

A subsample of $365612 \mathrm{~K}_{\mathrm{e} 3}^{0}$ events, from a larger number recorded by the CPLEAR experiment, was used to determine the parameter $\lambda_{+}$describing the linear energy dependence of the electroweak form factor $f_{+}\left(q^{2}\right)$. A ten times larger sample of events was simulated using Monte Carlo techniques. From the comparison of the real to simulated data samples, we obtained $\lambda_{+}=0.0245 \pm 0.0012_{\text {stat }} \pm 0.0022_{\text {syst }}$. When the errors are quadratically added, the total uncertainty is of the same order as in the best experiments reported up to now $[10,11]$. In addition no need for a quadratic term is found. Our result, when compared with the world average quoted in Ref. [2], namely $\lambda_{+}=0.0300 \pm 0.0016$, lies within 1.9 standard deviations of this value and leads to a new world average of $\lambda_{+}=0.0288 \pm 0.0014$.

\section{Acknowledgements}

We would like to thank the CERN LEAR staff for their support and co-operation as well as the technical and engineering staff of our institutes. This work was supported by the following agencies: the French CNRS/Institut National de Physique Nucléaire et de Physique des Particules, the French Commissariat à l'Energie Atomique, the Greek General Secretariat of Research and Technology, the Netherlands Foundation for Fundamental Research on Matter (FOM), the Portuguese National Board for Science and Technology (JNICT), the Ministry of Science and Technology of the Republic of Slovenia, the Swedish Natural Science Research Council, the Swiss National Science Foundation, the UK Particle Physics and Astronomy Research Council (PPARC), and the US National Science Foundation.

\section{References}

[1] L. Chounet et al., Phys. Reports 4 (1972) 199.

[2] C. Caso et al., Particle Data Group, Review of Particle Physics, Eur. Phys. J. C 3 (1998) 1.

[3] H.-M. Choi, C.-R. Ji, Phys. Rev. D 59 (1999) 034001, and references therein.

[4] A. Angelopoulos et al., CPLEAR Collaboration, Phys. Lett. B 444 (1998) 38.

[5] A. Angelopoulos et al., CPLEAR Collaboration, Phys. Lett. B 444 (1998) 43.

[6] A. Angelopoulos et al., CPLEAR Collaboration, Phys. Lett. B 444 (1998) 52.

[7] R. Adler et al., CPLEAR Collaboration, Nucl. Instrum. Methods A 379 (1996) 76.

[8] F. Blanc, PhD thesis (no. 1198), University of Fribourg, Switzerland, 1998.

[9] E. Ginsberg, Phys. Rev. 171 (1968) 1675, Errata in Phys. Rev. 174 (1968) 2169.

[10] G. Gjesdal et al., Nucl. Phys. B 109 (1976) 118.

[11] R. Blumenthal et al., Phys. Rev. Lett. 34 (1975) 164. 